\title{
China tackles surge in mental illness
}

\section{Psychological examinations to be added to selection procedure for government officials.}

\section{BY DAVID CYRANOSKI}

$\mathrm{I}$ $\mathrm{n}$ a country where the government likes to emphasize the harmony of its society and the well-being of its people, China's announcement on psychological examinations came as a surprise. A top official in the country's ruling Communist party said last month that the examinations will be introduced into the selection procedure for senior government officers and leaders of national industries. According to state media, the move was in part a response to a string of suicides by officials, many of whom suffered from depression.

It is the highest-level recognition yet of a problem that is widely affecting China's people, from the central arms of government to distant rural villages. According to a survey published last year, $17.5 \%$ of Chinese have some form of mental illness, one of the highest rates in the world (M. R. Phillips et al. Lancet 373, 2041-2053; 2009). In comparison, a World Health Organization survey found that the United States had the highest prevalence of mental illness in the world, with $26.4 \%$ of the population thought to have a diagnosable disorder in any given year (The WHO World Mental Health Survey Consortium J. Am. Med. Assoc. 291, 2581-2590; 2004).

In China, some blame the pressures of the social transformation accompanying the country's economic expansion; others argue that changes in the way mental illness is diagnosed might explain the rise. But with mental illness now firmly on the list of national health concerns, researchers, drug companies and medical practitioners are quickly developing new initiatives to tackle the issue.

\section{IDENTIFYING RISK}

Last year, the Chinese science ministry invested a total of 40 million yuan (US\$6 million) in ten two-year research projects that target issues such as prevention of suicide and depression, identifying early warning signs of the risk of developing mental illness, and dealing with occupational stress. For example, Wanjun Guo, a psychiatrist at the

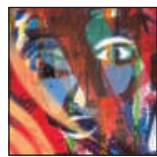

SCHIZOPHRENA

Search for origins and treatments nature.com/schizophrenia
Mental Health Center of West China Hospital in Sichuan, recently got 20 million yuan to "organize a survey on mental disorders in the 'post Wenchuan earthquake' region". Raymond Chan, a psychologist and deputy director of the Chinese Academy of Sciences Key Laboratory of Mental Health in Beijing, hopes that the funding will continue. He is seeking support for a databank of 'endophenotypes': characteristics that are thought to reflect a

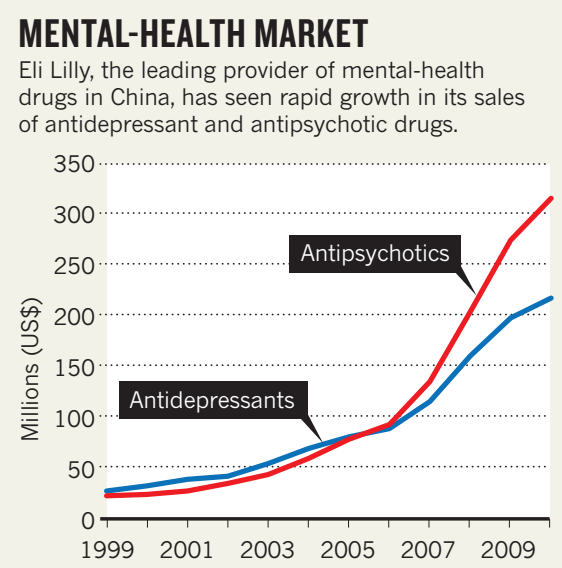

genetic disposition to mental diseases, such as eye-tracking dysfunction or memory failure in people with schizophrenia. "This will be a low cost, quick diagnostic tool," says Chan. "China probably needs it more than anywhere else."

China is poorly equipped to bring new findings into the clinic, however. Yanling $\mathrm{He}$, a psychiatrist at Shanghai Mental Health Center, says that there are only 20,000 psychiatrists in China: 1.5 for each 100,000 people, a tenth of the ratio in the United States. Many are poorly qualified, and they often deal with problems that would be handled by social workers or clinical psychologists elsewhere. Patchy distribution is an even greater worry, adds $\mathrm{He}$. Although there are 200 psychiatrists at the Shanghai Mental Health Center alone, the only practitioner in Tibet recently left, she says.

International collaborations are helping to strengthen China's mental-health infrastructure. Psychiatrists from the University of Melbourne in Australia have been working with the Chinese University of Hong Kong and the Peking University Institute of Mental Health to train people and establish communitybased health programmes, for example. The success of the collaboration has convinced China's health ministry to fund a scale-up to more than 100 sites, says University of Melbourne psychiatrist Chee $\mathrm{Ng}$, who helped to lead the effort. And Elise Snyder, a New Yorkbased psychoanalyst, is organizing the China American Psychoanalytic Alliance, which for the past two years has been training some of China's first psychotherapists through Skype conversations with US-based practitioners.

\section{DIAGNOSING CHANGE}

A change in government policy on medical insurance in 2005 also gave Chinese patients access to modern antidepressants and antipsychotics from abroad. "I see increased investment in mental-health infrastructure and wider coverage of essential treatment for patients," says Peter Salzmann, vice-president for marketing in China for pharmaceutical company Eli Lilly (see 'Mental-health market').

China's ministry of health has brought its mental-health guidelines and resources in line with international standards. The latest version of the Chinese Classification of Mental Disorders (CCMD), published in 2001, made Chinese definitions "nearly a replica" of the Diagnostic and Statistical Manual of Mental Disorders (DSM) issued by the American Psychiatric Association, according to Sing Lee, a psychiatrist at the Chinese University of Hong Kong.

But Lee worries that these changes may have led to the apparent boom in mental-health disorders. The latest CCMD follows the DSM in noting that depression can be diagnosed after just two weeks of symptoms - the previous duration recognized in China was three months, says Lee. "That will surely lead to high prevalence," he says. "It also increases the risk of medicalization of normal sadness like grief."

He adds that making discontent and unhappiness medical problems by focusing on psychological or psychiatric factors could distract attention from underlying social ills that might be to blame. Several of the government officials who committed suicide became depressed when they were investigated for corruption - a stark reminder that more psychiatrists and antidepressants alone might not be enough to cure China's mental-health woes. - 


\section{The roots of resistance}

\section{Learning how melanoma fights back may yield new therapies.}

\section{BY HEIDI LEDFORD}

A promising new cancer drug called PLX4032 made waves earlier this year for its success against the deadly cancer melanoma. The results of a clinical trial were dramatic, with some patients emerging nearly cancer-free. But for most, the drug stopped working about seven months after therapy began, and the tumours returned ${ }^{1}$. This week, two follow-up studies reveal why PLX4032 fails and how tumours' resistance to it might be overcome.

About half of patients with melanoma have a mutated version of the B-RAF protein, which is thought to help trigger the growth of tumour cells. PLX4032 was designed by Plexxikon, a pharmaceutical company in Berkeley, California, to selectively inhibit it. In one study ${ }^{2}$, PLX4032 shrank tumours in 24 of 32 patients with B-RAF mutations. But tumours often evolve ways to evade treatment, and this was no exception. Levi Garraway, an oncologist at the Dana-Farber
Cancer Institute in Boston, Massachusetts, and his colleagues have now found that excessive production of a cancer-promoting protein called COT can shield cultured cells from PLX4032. High levels of COT were also found in two of three PLX4032-resistant tumours taken from patients who had received the drug ${ }^{3}$.

A group led by Roger Lo, a dermatologist at the University of California, Los Angeles, found two other ways for tumour cells to short-circuit PLX4032: by reactivating the B-RAF signalling pathway damped down by the drug, or by stimulating tumour-cell growth mediated by a different protein ${ }^{4}$. Larger studies will be needed to assess the importance of both groups' results for clinical applications.

Still, the findings are a crucial step towards determining how to overcome PLX4032 resistance, says Alexis Borisy, chief executive of Foundation Medicine in Cambridge, Massachusetts, which is developing diagnostic tests for tumours. Pharmaceutical firms have already started clinical trials to test PLX4032 in combination with experimental drugs that inhibit MEK, a protein involved in cell growth. When Garraway's team tested this combination in their cultured tumour cells, they found that it overcame the effects of high levels of COT expression.

Clinicians may need to combine four or more drugs to create a potent cancer-fighting cocktail, says Borisy. PLX4032 is a good place to start, he notes: its highly selective targeting of B-RAF means it could have fewer unwanted side effects. "It's an exciting opportunity," he says.

1. Ledford, H. Nature 467, 140-141 (2010).

2. Flaherty, K. T. et al. N. Engl. J. Med. 363, 809-819 (2010).

3. Johannessen, C. M. et al. Nature advance online publication doi:10.1038/nature09627 (2010).

4. Nazarian, R. et al. Nature advance online publication doi:10.1038/nature09626 (2010).

\section{CORRECTION}

The News story 'China tackles surge in mental illness' (Nature 468, 145; 2010) incorrectly implied that Wanjun Guo leads a 20-million-yuan project to survey mental disorders in the "post Wenchuan earthquake' region. Guo is merely a participant in the project, which is itself only part of a 20-million-yuan effort to study the psychological impact of the quake and identify potential interventions. 